\section{RESEARCH LETTERS}

\section{Frontal fibrosing alopecia: epidemic?*}

\author{
Fabiane Mulinari Brenner ${ }^{1}$ \\ Carolina Oldoni ${ }^{2}$
}

DOI: http:/ / dx.doi.org/10.1590/abd1806-4841.20198300

\section{Dear Editor,}

Since the first description of frontal fibrosing alopecia (FFA) by Kossard in 1994, ${ }^{1}$ and its inclusion in the Consensus on Cicatricial Alopecia in 2001, this entity has been widely recognized by dermatologists. The first six cases described in Brazil, referred for publication in ABD in 2004 by our group, presented the classic description of postmenopausal frontal receding and eyebrow hairloss.

In the following years, descriptions of men and premenopausal women populated the literature and initiated the first debates regarding the disease. Recently, the involvement of facial, axillary and limb hair has changed the spectrum of the problem, suggesting systemic involvement of hair.

The association with autoimmune diseases such as thyroiditis, early ovarian failure and systemic lupus erythematosus suggests the influence of immunity on the pathogenesis of alopecia. The presence of other forms of lichen planus (LP), such as pigmented, ungual and mucous LP, prompted the exploration of agents inducing the lichenoid reaction pattern.

Since 2011, facial papules have been included among the characteristics of the disease. ${ }^{2}$ Lesions similar to rosacea, papules, comedones and facial pigmentation have been mistakenly addressed with aesthetic treatments such as peeling, LASER and pulsed light in recent years. Finally, aanother complication into the approach to these patients, suggested a possible association with the fre-

\footnotetext{
Received 15 March 2018

Accepted 27 June 2018

* Study conducted at the Hospital de Clínicas, Universidade Federal do Paraná, Curitiba, PR, Brazil.

Financial support: None.

Conflict of interest: None.

1 Dermatology Service, Hospital de Clínicas, Universidade Federal do Paraná, Curitiba, PR, Brazil.

2 Medical Student, Universidade Federal do Paraná, Curitiba, PR, Brazil.

MAILING ADDRESS:

Fabiane Mulinari Brenner

E-mail: fmbrenner@ufpr.br

C2019 by Anais Brasileiros de Dermatologia

quent use of cosmetics or sunscreen. ${ }^{3}$ Through a questionnaire that included several lifestyle, social and medical aspects, 105 women with FFA were compared to 100 healthy women who constituted the control group, paired by both sex and age. In addition, there was a subcohort of women who performed patch tests for various British standard allergens. The results showed significantly higher usage of sunscreen in the group of women with FFA, suggesting an association between this habit and the existence of FFA. There was also an increased frequency of positive contact tests and a higher incidence of thyroid disease in women with AFF, suggesting predisposition to immune-mediated diseases. The relationship between AFF and cosmetics is still only an assumption, but the warning of this possibility remains.

The initial group of 6 patients multiplied. Our database included 92 patients at the end of 2016 and doubled in 18 months (184 in 2018). New cases have been added monthly to this group, rising to 227 by march 2019. It is worth mentioning that this sample includes patients from a tertiary center of specialties and referrals. Recent cases present a new profile: younger patients with facial lesions and eyebrow or marginal alopecia that would easily be diagnosed as rosacea or atrophic pilar keratosis.

\section{REFERENCES}

1. Kossard S. Postmenopausal frontal fibrosing alopecia. Scarring alopecia in a pattern distribution. Arch Dermatol. 1994;130:770-4.

2. Donati A, Molina L, Doche I, Valente NS, Romiti R. Facial papules in frontal fibrosing alopecia: evidence of vellus follicle involvement. Arch Dermatol. 2011;147:1424-7.

3. Aldoori N, Dobson K, Holden CR, McDonagh AJ, Harries $M$, Messenger AG. Frontal fibrosing alopecia: possible association with leave-on facial skin care products and sunscreens; a questionnaire study. $\mathrm{Br} J$ Dermatol. 2016;175:762-7.
How to cite this article: Brenner FM, Oldoni C. Frontal Fibrosing Alopecia: epidemic? An Bras Dermatol. 2019;94(4):482. 